\section{Doenças sexualmente transmissíveis e gênero: um estudo transversal com adolescentes no Rio de Janeiro}

\author{
Sexually transmitted diseases and gender: \\ a cross-sectional study with adolescents \\ in Rio de Janeiro
}

Stella R. Taquette 1,2

Marília Mello de Vilhena 2

Mariana Campos de Paula 1

\footnotetext{
1 Faculdade de Ciências Médicas, Universidade do Estado do Rio de Janeiro, Rio de Janeiro, Brasil.

2 Núcleo de Estudos de Saúde do Adolescente, Universidade do Estado do Rio de Janeiro. Rio de Janeiro, Brasil.

Correspondência Stella R. Taquette, Faculdade de Ciências Médicas, Universidade do Estado do Rio de Janeiro. Av. Prof. Manoel de Abreu 444, Rio de Janeiro, $R J$ 20550-170, Brasil. taquette@uerj.br
}

\begin{abstract}
Sexually transmitted diseases (STDs) are frequent in adolescence and contribute to the increase in the number of AIDS cases. Early sexual initiation, multiple sex partners, and lack of condom use are considered risk factors and are influenced by a male-dominated gender system. We interviewed 356 adolescent patients at the Adolescent Health Research Center in Rio de Janeiro State University to identify possible STD risk factors in adolescence. Young men reported more partners and early sexual initiation. Females used condoms less frequently and were more subject to sexual abuse. The results confirm a model sustained by traditional gender values that demarcate the male and female spheres, with male supremacy. We conclude that to achieve more effective STD control, it is necessary to expand the discussion on culturally constructed patterns of masculinity and femininity.
\end{abstract}

Sexuality; Adolescence; Sexually Transmitted Diseases; Gender

\section{Introdução}

Na adolescência as relações sexuais têm iniciado mais cedo e com um maior número de parceiros, o que contribui para aumentar a ocorrência das DST 1,2. Entre adolescentes o uso de preservativos é baixo e a atividade sexual geralmente não é programada. Estudos brasileiros revelam que apenas um terço deles ou menos usam preservativo sempre $3,4,5$. Segundo dados de pesquisas divulgados pelo Ministério da Saúde (MS) 6, os mais baixos índices de uso se encontram entre 15 e 19 anos. No Brasil não há informações sobre a prevalência de DST entre adolescentes. As únicas DST de notificação compulsória são a sífilis e a AIDS e, além disso, cerca de $70 \%$ das pessoas com alguma DST buscam tratamento em farmácias, o que faz com que o número de casos notificados fique abaixo da estimativa 5 .

As DST favorecem a infecção pelo HIV. Do total de casos de AIDS, segundo as categorias de exposição, a via de transmissão sexual é a predominante (53\%) 7. De acordo com o MS 5, o maior número de notificações acumuladas entre 1980 e 1999 (67.267 casos, ou seja, 43,23\% do total) concentra-se entre 15 e 24 anos. Como o tempo de latência da doença é longo, chegando até 11 anos, podemos inferir que grande parte destes deve ter se infectado na adolescência 8,9 . Outra tendência da epidemia aponta para sua feminização e heterossexualização. 
Fatores biológicos, psíquicos, sociais, entre outros, podem interferir no desenvolvimento e expressão da sexualidade 10,11,12. O que é ser homem e/ou ser mulher tem suscitado inúmeras interpretações em diversos campos do saber. A atitude dos homens e das mulheres está intimamente ligada às representações simbólicas de masculinidade e feminilidade que se constróem historicamente, são mutáveis e relacionais.

A discussão das diferenças entre homem e mulher deu-se a partir da construção de uma nova imagem feminina política e econômica nos séculos XVIII e XIX. A emergência da polêmica cultural em torno da natureza e função da mulher na sociedade provocou uma nova ótica de significação da sexualidade humana 13. O conceito de gênero foi introduzido primeiramente nos Estados Unidos na década de 70 no campo da antropologia pelo movimento feminista. Este, ao longo dos tempos, foi se transformando e hoje não se pode afirmar que o feminismo contemporâneo seja igual ao do começo do século XIX 14. Considerando as distintas simbolizações de masculino e feminino no âmbito sexual, Garcia 15 afirma que gênero consiste na dimensão principal da construção social e dos significados que se relacionam ao sexo e à reprodução, varia de uma cultura para outra, em diferentes tempos históricos e também ao longo da vida de um indivíduo. Segundo Strey 14, psicóloga social, gênero não é sexo. Enquanto este último diz respeito às características físicas relativas à reprodução biológica, o primeiro consiste numa construção cultural que depende de como a sociedade transforma um macho num homem e uma fêmea numa mulher. A análise da vida sexual envolve a dupla referência dos impulsos biológicos e da regulamentação social 16. Daí o enfoque de gênero numa perspectiva relacional e transversal que interage com classe social, raça/etnia, diferenças de geração, cultura etc. O gênero, portanto, é um sistema que atua no campo social por vezes de modo contraditório para mulheres e homens em variadas situações 17,18.

A análise do gênero contribuiu enormemente na discussão da sexualidade nas últimas décadas por meio da crítica ao determinismo biológico, suporte da supremacia masculina 19. Apesar da ocorrência de grandes transformações no século passado nas relações entre os gêneros pelas conquistas das mulheres (entrada no mercado de trabalho, direito ao voto, maior escolaridade e separação entre sexualidade e reprodução) ainda hoje persiste um quadro de "dominação masculina" 20. Atualmente no ocidente, a tradição patriarcal identifica o masculino com razão, regulação, liberdade de exercício sexual e conhecimento da sexualidade feminina 21,22 . Já a mulher é marcada fortemente pela inexperiência sexual, pelo ocultamento, silêncio, conformismo 23. O gênero imprime normas, valores, percepções, representações que acompanham a vida do sujeito legitimando sua identidade. Apesar da complexidade e pluralidade das identidades subjetivas, a dominação dos sistemas de gênero em sua lógica de poder pode resvalar numa naturalização que justifica as desigualdades sociais entre homens e mulheres 24 . Foucault 25 considera a sexualidade como dispositivo de poder fortemente impregnado por relações assimétricas entre homens e mulheres que faz aparecer de forma contundente as relações entre o biológico e o social.

Observamos em pesquisa anterior realizada com adolescentes dos bairros de Vila Isabel e Acari a assimetria referida acima. Nesta, o modelo de dominação de gênero masculino foi um dos principais geradores de violência e risco de DST, ao dificultar a negociação do uso do preservativo entre os parceiros em suas relações sexuais 26. Muszkat 27 verificou em estudo com 2 mil famílias atendidas no Pró-Mulher, Família e Cidadania um forte elo entre virilidade e violência nas camadas mais pobres da população. Para D'Oliveira, 28 as raízes da violência nas relações de gênero encontram-se nas próprias relações de gênero. Os homens cedo necessitam provar que são potentes e sexualmente capazes, enquanto as mulheres são passivas, dependentes e sensíveis.

A naturalização deste modelo de gênero pode causar impacto à saúde 29 , pois para cumprir os padrões do que é ser homem e/ou mulher, ambos têm de desempenhar uma prática sexual que lhes é nociva do ponto de vista do risco às DST e à própria vida. Por um lado, os homens lançam-se em situações perigosas e freqüentemente recorrem à violência contra sua parceira por desejo de poder e controle. Por outro lado, as mulheres a eles se submetem, muitas vezes em detrimento da própria saúde, como no caso das relações sexuais em que seu parceiro se recusa a usar o preservativo. Os jovens se mostram insatisfeitos com os papéis de gênero a desempenhar. As consultas devido a dificuldades de ereção, ejaculação precoce, necessidade de bebidas alcoólicas para se consumar o ato sexual e preocupações 
com o tamanho do pênis são freqüentes. As moças, por sua vez, sentem-se pressionadas pelo grupo a iniciar a atividade sexual, pelo namorado para dar "provas de amor" mediante concessão ao coito e não conseguem se impor na negociação do uso do preservativo.

O objetivo do presente estudo foi conhecer a população adolescente atendida no Núcleo de Estudos em Saúde do Adolescente (NESA), da Universidade do Estado do Rio de Janeiro (UERJ), do ponto de vista da sexualidade e descrever possíveis fatores de risco às DST. O trabalho apresenta e discute estes fatores relacionando-os a um modelo de sistema de gênero que supõe uma supremacia masculina.

\section{População estudada e método}

Trata-se de um estudo observacional, do tipo transversal cuja população alvo foi o público adolescente que procurou atendimento médico no NESA entre agosto de 2001 e julho de 2002. O NESA é uma instituição pública cujo ambulatório atende adolescentes de 12 a 19 anos, a maioria deles pertencente às classes de baixo poder aquisitivo, em diversas especialidades.

A amostra estudada foi de conveniência e a escolha dos participantes foi aleatória (não probabilística) entre os adolescentes que aguardavam atendimento em sala de espera. Desconhecia-se de antemão o motivo da consulta e se o adolescente era ou não sexualmente ativo. $\mathrm{O}$ instrumento utilizado foi uma entrevista semi-estruturada que obedecia a um roteiro previamente estabelecido e testado por um estudo piloto com vinte adolescentes, não incluídos na amostra do estudo. Todos os entrevistadores receberam treinamento por um único pesquisador antes de ir ao campo e semanalmente eram feitas reuniões da equipe de pesquisa onde os dados eram checados. A validade das informações foi assegurada de várias maneiras. Quando havia dúvida quanto à sua veracidade, o participante era excluído da amostra. Além disso, cerca de $5 \%$ das entrevistas foram repetidas por outro entrevistador que obteve as mesmas respostas. Cada pesquisador entrevistou uma proporção semelhante de homens, mulheres e portadores ou não de DST. Estes procedimentos foram efetuados no sentido de garantir uma homogeneidade interna.

As entrevistas eram realizadas a sós com o adolescente, após consentimento informado. Elas foram sendo realizadas sucessivamente até completar-se cem casos de DST, o que ocorreu doze meses após seu início. Critérios para inclusão e razões para escolha desta amostra- gem: privilegiamos de modo intencional os ambulatórios de DST, ginecologia e urologia, onde a possibilidade de existirem pacientes sexualmente ativos e portadores de DST era maior. Concomitantemente adolescentes eram entrevistados nos outros ambulatórios.

Os casos de DST foram diagnosticados por critério clínico e/ou laboratorial, utilizando-se a abordagem sindrômica definida no manual de DST do MS 30 e testes diagnósticos específicos. Considerou-se no diagnóstico todas as patologias que podem ser transmitidas por meio do ato sexual, incluindo aquelas que são essencialmente assim transmitidas (sífilis, gonorréia e o HPV), freqüentemente (uretrites não gonocócicas, candidíase, tricomoníase, herpes simples, hepatite B e o HIV) e eventualmente (escabiose, fitiríase), desde que houvesse sintomas clínicos evidentes e/ou exames positivos e relatos da anamnese que garantissem a transmissão sexual da doença 31 .

O roteiro de entrevista, composto de três partes, contava com perguntas abertas e fechadas. A primeira investigava dados pessoais como idade, renda familiar, escolaridade, trabalho, prática de exercícios, uso de bebidas alcoólicas, tabaco e outras drogas. Considerou-se atraso escolar uma defasagem maior que dois anos em relação à idade esperada para a série freqüentada. Sobre a prática de exercícios, esta era considerada regular quando no mínimo três vezes por semana. O uso de álcool e de drogas foi classificado em uma vez na vida, no último mês ou seis vezes ou mais no último mês.

Na segunda parte da entrevista perguntamos detalhadamente sobre a família: com quem morava, opinião sobre o pai, a mãe, seu relacionamento com cada um dos genitores e entre eles. Estas perguntas eram abertas e o entrevistador cuidadoso no sentido de não sugestionar as respostas, como, por exemplo, perguntar se o pai era bom.

$\mathrm{Na}$ terceira parte investigou-se o histórico pubertário e sexual do adolescente, a época da menarca/semenarca e da primeira relação sexual. A respeito do primeiro coito, com quem ocorreu, se com namorado/a, amigo/a ou profissional do sexo; se a relação sexual aconteceu de forma espontânea (com consentimento do entrevistado), por pressão (quando o entrevistado foi coagido/a) ou forçada (à revelia do entrevistado). Em seguida, indagamos quanto à ocorrência de homossexualismo, prostituição, abuso sexual e gravidez. As últimas perguntas foram sobre o número de parceiros sexuais; uso de preservativos, se nunca, às vezes, quase sempre ou sempre.

$\mathrm{Na}$ análise estatística das respostas às perguntas estruturadas utilizamos o teste Qui-qua- 
drado com nível de significância de $95 \% 32$. As respostas às perguntas abertas foram lidas e relidas exaustivamente e a partir disso foram construídas categorias, depois quantificadas e analisadas estatisticamente.

O projeto de pesquisa foi previamente avaliado e autorizado pelo Comitê de Ética em Pesquisa do Hospital Universitário Pedro Ernesto e cumpre os princípios éticos contidos na Declaração de Helsinki. O termo de consentimento livre e esclarecido foi assinado antes da entrevista.

\section{Resultados}

Todas as entrevistas foram realizadas em ambiente com privacidade. O tempo médio de duração de cada uma foi de 25 minutos. No total foram entrevistados 356 adolescentes. Entre estes 109 eram sexualmente ativos portadores de DST, 115 sexualmente ativos porém sem DST e 132 ainda não tinham iniciado atividade sexual. Adolescentes do sexo masculino compunham $29,5 \%$ da amostra e do sexo feminino, $70,5 \%$. O predomínio de moças ocorreu devido ao grande volume de atendimento do ambulatório de ginecologia. O percentual de homens e mulheres em cada grupo foi semelhante. Estes dados podem ser visualizados na Tabela 1 .

A idade média do grupo masculino foi de 16,5 anos e do feminino 16,4. Todos os adolescentes pertenciam às camadas sociais de baixo poder aquisitivo. Os dados referentes à idade, situação sócio-econômica, escolaridade, trabalho e atividade física se encontram na Tabela 2. Foram significativas as associações entre os homens e as variáveis: atraso escolar maior que dois anos e atividade física regular.

Na Tabela 3 estão descritos os dados referentes ao uso de tabaco, álcool e drogas. Em relação ao consumo de bebidas alcoólicas, a maioria dos adolescentes já tinha feito uso pelo menos uma vez na vida. Entre os que usavam ou já tinham feito uso de drogas pelo menos uma vez na vida, observou-se um percentual significativamente maior de homens.

Em relação à família, cerca de metade dos adolescentes vivia com ambos os pais e $28,9 \%$ com apenas um dos genitores, sendo na maioria deles a mãe $(8,7 \%$ pai e $91,3 \%$ mãe). Houve um predomínio de depoimentos positivos a respeito das mães. Segundo a opinião dos entrevistados, $45,5 \%$ de seus pais e $73,9 \%$ de suas mães tinham qualidades.

Na Tabela 4 estão descritos os dados referentes à sexualidade. Do total dos entrevistados, 224 eram sexualmente ativos, sendo $28,1 \%$ do sexo masculino e $71,9 \%$ do feminino. Dentre estes, a idade média da semenarca foi 13 anos e da menarca 12 anos. A primeira relação sexual ocorreu em média aos 14,6 anos e aos 15 entre os rapazes e moças, respectivamente. $\mathrm{O}$ tempo médio decorrido entre a menarca/semenarca e o primeiro coito foi de 1,6 anos entre os rapazes e 2,9 anos entre as moças. Mais da metade dos adolescentes praticou o primeiro coito até um ano após a semenarca e em menos de um quarto das adolescentes ocorreu o mesmo em relação à menarca, diferença estatisticamente significativa. O primeiro intercurso sexual aconteceu com namorado/a para $33,3 \%$ dos adolescentes e $90,7 \%$ das adolescentes. Por outro lado, $55,5 \%$ dos rapazes iniciaram o sexo com amigas e apenas $9,3 \%$ das moças fizeram o mesmo. Nenhuma adolescente teve o primeiro intercurso com profissionais do sexo e $11,7 \%$ dos rapazes relataram esta prática. O sexo ocorreu de forma espontânea para a maioria. O histórico de gravidez e de abuso sexual foi significativamente mais freqüente entre as moças. Nos relatos de abuso sexual, exceto um, o perpetrador era do sexo masculino. A prostituição foi maior entre os homens, assim como as relações homossexuais. O número de parceiros foi maior do que dois para $71,4 \%$ dos homens e $28,6 \%$ das mulheres, relação estatisticamente significativa. O uso de preservativo nas relações sexuais foi significativamente menos freqüente entre as moças.

Os diagnósticos mais comuns nos homens foram as uretrites e nas mulheres as vulvovaginites. As uretrites foram confirmadas por exames laboratoriais. Nas vulvovaginites o diagnóstico foi predominantemente clínico. Não foi possível a coleta de secreção vaginal em todos os casos. $\mathrm{O}$ diagnóstico da sífilis foi feito pelo quadro/história clínica e exame VDRL. Nos casos de HPV e de herpes genital o diagnóstico foi clínico. Houve quatro casos de HIV+ por transmissão sexual, com diagnóstico sorológico realizado obedecendo-se ao fluxograma preconizado pelo MS 30, num adolescente masculino e em três femininos. Verificamos um caso de hepatite B com IgM positiva (Anti-HbcIgM) em um paciente com gonorréia, sem histórico de uso de injeções ou transfusões sangüíneas. Houve três casos de escabiose em que o diagnóstico foi feito por meio dos sintomas clínicos e teste terapêutico, após terem sido afastados outros diagnósticos utilizando-se exames laboratoriais. Em alguns casos houve concomitância de mais de uma DST. Na Tabela 5 optou-se por descrever somente a distribuição dos diagnósticos principais de DST. 
Distribuição dos participantes segundo presença/ausência de atividade sexual e DST.

\begin{tabular}{|c|c|c|c|c|c|c|c|c|}
\hline \multirow[t]{2}{*}{ Grupo } & \multicolumn{2}{|c|}{ Amostra total } & \multicolumn{2}{|c|}{$\begin{array}{l}\text { Sexualmente } \\
\text { ativos com DST }\end{array}$} & \multicolumn{2}{|c|}{$\begin{array}{l}\text { Sexualmente } \\
\text { ativos sem DST }\end{array}$} & \multicolumn{2}{|c|}{$\begin{array}{l}\text { Sem atividade } \\
\text { sexual }\end{array}$} \\
\hline & $\mathrm{n}$ & $\%$ & $n$ & $\%$ & $n$ & $\%$ & $n$ & $\%$ \\
\hline Homens & 105 & 29,5 & 31 & 28,4 & 32 & 27,8 & 42 & 31,8 \\
\hline Mulheres & 251 & 70,5 & 78 & 71,6 & 83 & 72,2 & 90 & 68,2 \\
\hline Total & 356 & 100,0 & 109 & 100,0 & 115 & 100,0 & 132 & 100,0 \\
\hline
\end{tabular}

Tabela 2

Distribuição dos participantes segundo sexo e características demográficas.

\begin{tabular}{|c|c|c|c|c|c|c|}
\hline Item pesquisado & \multicolumn{2}{|c|}{ Homens } & \multicolumn{2}{|c|}{ Mulheres } & $\begin{array}{l}\text { Qui- } \\
\text { quadrado }\end{array}$ & $\mathbf{P}$ \\
\hline Idade média (em anos) & \multicolumn{2}{|c|}{16,5} & \multicolumn{2}{|c|}{16,4} & & \\
\hline \multirow[t]{2}{*}{ Renda familiar (em salários mínimos) } & \multicolumn{2}{|c|}{4,4} & \multicolumn{2}{|c|}{3,9} & & \\
\hline & n (105) & $\%$ & n (251) & $\%$ & & \\
\hline \multicolumn{7}{|l|}{ Histórico escolar } \\
\hline estão na escola & 87 & 82,8 & 212 & 84,5 & 0,05 & 0,82 \\
\hline atraso escolar $>2$ anos & 43 & 40,9 & 72 & 28,7 & 4,55 & 0,03 \\
\hline Atividade física regular & 41 & 39,0 & 28 & 11,2 & 35,10 & 0,00 \\
\hline Trabalham & 28 & 26,7 & 46 & 18,3 & 2,64 & 0,10 \\
\hline
\end{tabular}

Tabela 3

Distribuição dos participantes segundo sexo e o uso de bebidas alcoólicas, tabaco e outras drogas.

\begin{tabular}{|c|c|c|c|c|c|c|}
\hline \multirow[t]{2}{*}{ Item pesquisado } & \multicolumn{2}{|c|}{ Homens } & \multicolumn{2}{|c|}{ Mulheres } & \multirow{2}{*}{$\begin{array}{l}\text { Qui- } \\
\text { quadrado }\end{array}$} & \multirow[t]{2}{*}{$p$} \\
\hline & n (105) & $\%$ & $\mathrm{n}(251)$ & $\%$ & & \\
\hline \multicolumn{7}{|l|}{ Uso de bebidas alcoólicas } \\
\hline Nunca usou & 32 & 30,5 & 78 & 31,1 & 0,00 & 0,98 \\
\hline Usou uma vez na vida & 32 & 30,5 & 95 & 37,8 & 1,45 & 0,22 \\
\hline Usou no último mês & 32 & 30,5 & 67 & 26,7 & 0,36 & 0,55 \\
\hline Usou 6 vezes no último mês & 9 & 8,5 & 11 & 4,4 & 1,72 & 0,18 \\
\hline \multicolumn{7}{|l|}{ Uso de tabaco } \\
\hline Sim & 12 & 11,4 & 32 & 12,7 & 0,03 & 0,86 \\
\hline Não & 93 & 88,6 & 219 & 87,3 & & \\
\hline \multicolumn{7}{|l|}{ Uso de outras drogas } \\
\hline Sim & 24 & 22,9 & 17 & 6,8 & 17,25 & 0,00 \\
\hline Não & 81 & 77,1 & 234 & 93,2 & & \\
\hline
\end{tabular}




\begin{tabular}{|c|c|c|c|c|c|c|}
\hline \multirow[t]{2}{*}{ Item pesquisado } & \multicolumn{2}{|c|}{ Homens } & \multicolumn{2}{|c|}{ Mulheres } & \multirow{2}{*}{$\begin{array}{l}\text { Qui- } \\
\text { quadrado }\end{array}$} & \multirow[t]{2}{*}{$\mathbf{P}$} \\
\hline & n (63) & $\%$ & $\mathrm{n}(161)$ & $\%$ & & \\
\hline $\begin{array}{l}\text { Idade média da menarca/ } \\
\text { semenarca (em anos) }\end{array}$ & 13 & & 12 & & & \\
\hline \multicolumn{7}{|l|}{ Características do 1 o coito } \\
\hline Idade média (em anos) & 14,6 & & 15 & & & \\
\hline $\begin{array}{l}\text { Tempo médio entre a menarca/ } \\
\text { semenarca e o 1ㅇ coito (em anos) }\end{array}$ & 1,6 & & 2,9 & & & \\
\hline $\begin{array}{l}\text { Menos de } 1 \text { ano após } \\
\text { a menarca/semenarca }\end{array}$ & 35 & 55,5 & 39 & 24,2 & 18,70 & 0,00 \\
\hline \multicolumn{7}{|l|}{ Tipo de parceiro/a no 1o coito } \\
\hline Namorado/a & 21 & 33,3 & 146 & 90,7 & 75,51 & 0,00 \\
\hline Amigo/a & 35 & 55,5 & 15 & 9,3 & 53,20 & 0,00 \\
\hline Profissional do sexo & 7 & 11,7 & 0 & 0,0 & 14,98 & 0,00 \\
\hline \multicolumn{7}{|l|}{ Como aconteceu o 1 o coito } \\
\hline Espontâneo & 61 & 96,8 & 152 & 94,4 & 0,17 & 0,68 \\
\hline Forçado & 0 & 0,0 & 2 & 1,2 & 0,01 & 0,92 \\
\hline Por pressão & 2 & 3,2 & 7 & 4,3 & 0,00 & 0,98 \\
\hline Histórico de gravidez & 8 & 12,7 & 42 & 26,1 & 3,94 & 0,04 \\
\hline Histórico de abuso & 6 & 9,5 & 42 & 26,1 & 6,43 & 0,01 \\
\hline Histórico de prostituição & 10 & 15,9 & 5 & 3,1 & 9,86 & 0,00 \\
\hline Homossexualismo & 12 & 19,0 & 1 & 0,6 & 24,85 & 0,00 \\
\hline Número de parceiros $>2$ & 45 & 71,4 & 46 & 28,6 & 32,73 & 0,00 \\
\hline \multicolumn{7}{|l|}{ Uso de preservativo } \\
\hline Nunca ou às vezes & 29 & 46,0 & 112 & 64,5 & & \\
\hline Quase sempre ou sempre & 34 & 54,0 & 49 & 35,5 & 9,77 & 0,00 \\
\hline
\end{tabular}

\section{Discussão}

Por meio da análise dos dados descritos constatamos que algumas diferenças entre homens e mulheres foram significativas e corroboram achados de outros autores. A menor idade de iniciação sexual entre os rapazes foi verificada por Scivoletto 33, Anteghini 34 e Warren 35. O MS 6 destaca que as mulheres usam menos preservativos do que os homens, enquanto Siegel 36, em estudo com 797 estudantes universitários americanos, assinala que as mulheres têm menor número de parceiros.

Os papéis desempenhados pelos adolescentes estudados se incluem num modelo de dominação de gênero no qual a visão de masculinidade e feminilidade dificulta o avanço de programas de promoção à saúde. Em nossa pesquisa, os adolescentes masculinos praticam mais regularmente atividades físicas, usam drogas com maior freqüência, entram em ativida- de sexual mais cedo com amigas, profissionais do sexo, "transam" com homossexuais, têm mais parceiros/as e se prostituem mais. Quanto às moças, estas privilegiam relações afetivas (namorados), são vítimas mais freqüentes de abuso sexual e usam menos preservativos.

Nesta hierarquia de gênero, a supremacia masculina refere-se à força, violência, virilidade, "macho potente" que se prostitui, "transa" até com homens 37 e não nega qualquer relação sexual. Ser homem neste contexto significa soberania, repudiar o feminino, não revelar sentimentos e arriscar sempre, mesmo por meio de violência 15 . Ser mulher, então, associa-se à passividade frente ao abuso sexual, submissão, cuidado e temor em relação ao homem, enfim, resignação ao papel cristalizado de objeto do controle masculino 38. A desigualdade de poder nas relações entre homens e mulheres é um dos motivos da dificuldade que ambos têm em discutir formas seguras de exercer a sexualidade 39. 
Tabela 5

Distribuição dos participantes segundo sexo e diagnóstico de DST.

\begin{tabular}{lrrrr}
\hline Diagnóstico principal & \multicolumn{2}{c}{ Homens } & \multicolumn{2}{c}{ Mulheres } \\
& $\mathrm{n}(31)$ & $\%$ & $\mathrm{n}(78)$ & $\%$ \\
\hline Uretrites gonocócicas e não gonocócicas & 17 & 54,8 & 3 & 3,8 \\
Vulvovaginites (candidíase, vaginose & 0 & 0,0 & 47 & 60,3 \\
e tricomoníase) & & & & \\
HPV & 7 & 22,6 & 15 & 19,2 \\
Sífilis & 3 & 9,7 & 7 & 9,0 \\
HIV+ & 1 & 3,2 & 3 & 3,8 \\
Herpes genital & 0 & 0,0 & 2 & 2,6 \\
Escabiose & 2 & 6,5 & 1 & 1,3 \\
Hepatite B & 1 & 3,2 & 0 & 0,0 \\
\hline
\end{tabular}

Promover a saúde humana, reduzir a incidência de DST em adolescentes inclui, certamente, como pudemos constatar mediante os resultados desta pesquisa, a ampliação do debate em torno da existência de modelos de dominação de gênero. A principal via de transmissão da AIDS é a sexual e o maior número de casos notificados ao MS incide em homens heterossexuais que, apesar de possuírem variadas parceiras, não se sentem ameaçados pela epidemia e nem pertencentes a um grupo de risco. Deste modo, a vulnerabilidade da mulher aumenta, pois lhe falta o poder de negociação e controle sobre suas relações sexuais em termos de fidelidade mútua e uso de preservativo pelo homem 40 .
A necessidade de maior compreensão de novos significados das relações entre homens e mulheres é ressaltada por Arilha 41 . Os papéis de gênero desempenhados pelo homem e pela mulher podem e devem ser modificados na medida em que paralisam os indivíduos em seus desejos e conquistas. Talvez esteja faltando um olhar mais extenso em relação às suas singularidades para que ambos se enriqueçam com elas.

Quanto aos adolescentes por nós atendidos no NESA, desejamos que não se submetam pura e simplesmente a padrões de gênero impostos como imutáveis. Nossas ações de saúde devem inseri-los em atividades, como, por exemplo, discussões em grupo seriadas em que haja um espaço de escuta e troca de informações que os ajudem a refletir sobre seus papéis e a ser participantes ativos na construção de sua sexualidade. As estratégias de informação devem ser diferenciadas para cada um dos gêneros, como destacam alguns autores $42,43,44,45$, já que o sentido que a mulher e o homem dão ao corpo sexual e reprodutivo é distinto.

Vale lembrar que este estudo empregou um processo de amostragem de conveniência. Portanto, os dados referem-se a grupos específicos de adolescentes que não podem ser generalizados para toda a população adolescente do Rio de Janeiro. Entretanto, nossos resultados nos auxiliam no entendimento e a pensar na problemática das DST na adolescência e são semelhantes aos encontrados em outras pesquisas 46,47 .

\section{Resumo}

As doenças sexualmente transmissíveis (DST) são freqüentes na adolescência e podem contribuir no aumento do número de casos de AIDS. A iniciação sexual precoce, a multiplicidade de parceiros e o não uso de preservativo nas relações sexuais têm sido apontados como fatores de risco, e são influenciados por um sistema de gênero que se pauta na dominação masculina. Para identificar possiveis fatores de risco às DST na adolescência, foi feito um estudo com 356 adolescentes atendidos no Núcleo de Estudos da Saúde do Adolescente da Universidade do Estado do Rio de Janeiro, por meio de entrevistas semi-estruturadas. Os principais resultados na população estudada mostraram que os rapazes têm maior número de parcei- ras e iniciam a atividade sexual mais cedo. As moças, por sua vez, usam menos preservativo e são vítimas mais freqüentes de abuso sexual. Estes dados confirmam um modelo sustentado em valores tradicionais de gênero que demarcam as esferas masculina e feminina supondo uma supremacia da primeira.Concluise que para se ter um controle mais efetivo das DST é necessário ampliar-se o debate em torno dos modelos de masculinidade e feminilidade culturalmente construídos.

Sexualidade; Adolescência; Doenças Sexualmente Transmissíveis;Gênero 


\section{Colaboradores}

A contribuição dos autores na elaboração do artigo se deu de forma eqüitativa em todas as etapas do processo; incluindo coleta de dados, análise e redação do manuscrito.

\section{Referências}

1. Petri V. Doenças de transmissão sexual. In: Comissão de Saúde do Adolescente, Secretaria Estadual de Saúde de São Paulo, organizador. Adolescência e saúde. São Paulo: Paris Editorial; 1988. p. 183-90.

2. Waystaff DA, Delameth JD, Havens KK. Subsequent infection among adolescent African-American males attending a sexually transmitted disease clinic. J Adolesc Health 1999; 25:217-26.

3. Jiménez AL, Gotlieb SLD, Hardy E, Zaneveld LJD. Prevenção de doenças sexualmente transmissíveis em mulheres: associação com variáveis socioeconômicas e demográficas. Cad Saúde Pública $2001 ; 17: 55-62$.

4. Trajman A, Belo MT, Teixeira EG, Dantas VCS, Salomão FM, Cunha AJLA. Knowledge about STD/ AIDS and sexual behavior among high school students in Rio de Janeiro, Brazil. Cad Saúde Pública 2003; 19:127-33.

5. Ministério da Saúde. Dados e pesquisas em DST e Aids. http://www.aids.gov.br (acessado em 24/ Abr/2003).

6. Ministério da Saúde. Preservativo masculino. Brasília: Secretaria de Assistência à Saúde; 1997.

7. Castilho EA, Szwarcwald CL. Mais uma pedra no meio do caminho dos jovens brasileiros: a AIDS. In: Comissão Nacional de População e Desenvolvimento, organizador. Jovens acontecendo na trilha das políticas públicas, Brasília: Comissão Nacional de População e Desenvolvimento; 1998. p. 197-207.

8. Santos VL, Santos CE. Adolescentes, jovens e Aids no Brasil. In: Ministério da Saúde, organizador. Cadernos Juventude Saúde e Desenvolvimento I. Brasília: Ministério da Saúde; 1999. p. 213-22.

9. Chequer P. A AIDS no Brasil: perfil epidemiológico e ações. Folha Med 1998; 117:1-2.

10. Bozon M. Sobre campanhas de comunicação... Saúde em Foco 1998; 17:20.

11. Bastos FI, Carlini-Contrin B. O consumo de substâncias psicoativas entre os jovens brasileiros: dados, danos \& algumas propostas. In: Comissão Nacional de População e Desenvolvimento, organizador. Jovens acontecendo na trilha das políticas públicas. Brasília: Comissão Nacional de População e Desenvolvimento; 1998. p. 645-69.

12. Arthur L, Whaley MPH. Preventing the high-risk sexual behavior of adolescents: focus on HIV/AIDS transmission, unintended pregnancy, or both? J Adolesc Health 1999; 24:376-82.

\section{Agradecimentos}

Agradecemos a participação, durante o desenvolvimento da pesquisa, da médica ginecologista Dra. Renata Bessa de Andrade, dos seguintes alunos bolsistas de graduação da UERJ: Felipe K. dos Santos, Felipe Nirenberg, Ene Garcez Neto e Úrsula Pérsia P. Santos.

13. Costa JF. O referente da identidade homossexual. In: Parker R, Barbosa RM, organizadores. Sexualidades brasileiras. Rio de Janeiro: Relume Dumará; 1996. p. 63-89.

14. Strey MN. Gênero. In: Guareschi P, organizador. Psicologia social contemporânea. Petrópolis: Vozes; 1998. p. 181-98.

15. Garcia SM. Conhecer os homens a partir do gênero e para além do gênero. In: Arilha M, Ridenti GU, Medrado B, organizadores. Homens e masculinidades. Outras palavras. São Paulo: Editora 34; 1998. p. 31-50.

16. Loyola MA. Sexo e sexualidade na antropologia. In: Loyola MA, organizador. A sexualidade nas ciências humanas. Rio de Janeiro: Eduerj; 1998. p. 17-47.

17. Villela W. Sobre sexo e sexualidade em tempos de AIDS. Saúde em Foco 1998; 7:17-9.

18. Giffin K. Pobreza, desigualdade e eqüidade em saúde: considerações a partir de uma perspectiva de gênero transversal. Cad Saúde Pública 2002; 18 Suppl:103-12.

19. Barbosa RHS. Aids e saúde reprodutiva: novos desafios. In: Giffin K, Costa H, organizadores. Questões da saúde reprodutiva. Rio de Janeiro: Editora Fiocruz; 1999. p. 281-98.

20. Heilborn ML. Construção de si, gênero e sexualidade. In: Heilborn ML, organizador. Sexualidade: o olhar das ciências sociais. Rio de Janeiro: Jorge Zahar; 1999. p. 40-58.

21. Corrêa S. Gênero e sexualidade como sistemas autônomos: idéias fora do lugar? In: Parker R, Barbosa RM, organizadores. Sexualidades brasileiras. Rio de Janeiro: Relume Dumará; 1996. p. 149-59.

22. Giffin K. Corpo e conhecimento na saúde sexual: uma visão sociológica. In: Giffin $\mathrm{K}$, Costa $\mathrm{H}$, organizadores. Questões da saúde reprodutiva. Rio de Janeiro: Editora Fiocruz; 1999. p. 79-94.

23. Guimarães K. Nas raízes do silêncio: a representação cultural da sexualidade feminina e a prevenção do HIV/AIDS. In: Parker R, Galvão J, organizadores. Quebrando o silêncio mulheres e AIDS no Brasil. Rio de Janeiro: Relume Dumará; 1996. p. 89-113.

24. Corrêa S. "Saúde reprodutiva", gênero e sexualidade: legitimação e novas interrogações. In: Giffin K, Costa H, organizadores. Questões da saúde reprodutiva. Rio de Janeiro: Editora Fiocruz; 1999. p. 39-50.

25. Foucault M. História da sexualidade I - a vontade de saber. Rio de Janeiro: Graal; 1988. 
26. Taquette SR, Ruzany MH, Meirelles Z, Ricardo I. Relacionamento violento na adolescência e risco de DST/AIDS. Cad Saúde Pública 2003; 19:143744.

27. Muszkat ME. Violência de gênero e paternidade. In: Arilha M, Ridenti SGU, Medrado B, organizadores. Homens e masculinidades. Outras palavras. São Paulo: Editora 34; 1998. p. 215-58.

28. D'Oliveira AFPL, Schraiber LB. Violência de gênero, saúde reprodutiva e serviços. In: Giffin K, Costa H, organizadores. Questões da saúde reprodutiva. Rio de Janeiro: Editora Fiocruz; 1999. p. 33756.

29. Korin D. Nuevas perspectivas de género en salud. Adolescencia Latinoamericana 2001; 2:67-79.

30. Ministério da Saúde. Manual de controle de doenças sexualmente transmissíveis. Brasília: Secretaria de Políticas de Saúde; 1999.

31. Azulay DR, Manela-Azulay M. Doenças sexualmente transmissíveis. In: Schechter M, Marangoni DV, organizadores. Doenças infecciosas: conduta diagnóstica e terapêutica. Rio de Janeiro. Guanabara Koogan; 1998. p. 467-88.

32. Arango HG. Bioestatística teórica e computacional. Rio de Janeiro: Guanabara Koogan; 2001.

33. Scivoletto S, Tsuji RK, Abdo CHN, Queiroz S, Andrade AG, Gattaz WF. Relação entre consumo de drogas e comportamento sexual de estudantes de 2o grau de São Paulo. Rev Bras Psiquiatr 1999; 21:87-94.

34. Anteghini M, Fonseca H, Ireland M, Blum RW. Health risk behaviors and associated risk and protective factors among Brazilian Adolescents in Santos, Brazil. J Adolesc Health 2001; 28:295302 .

35. Warren CW, Kann L, Small ML, Santelli JS, Collins JL, Kolbe LJ. Age of initiating selected health-risk behaviors among high school students in the United States. J Adolesc Health 1997; 21:225-31.

36. Siegel DM, Klein DI, Roghmann KJ. Sexual behavior, contraception and risk among college students. J Adolesc Health 1999; 25:336-43.

37. Picazio C. Sexo secreto. São Paulo: Summus Editorial; 1999.
38. Mota MP. Gênero e sexualidade: fragmentos de identidade masculina nos tempos de AIDS. Cad Saúde Pública 1998; 14:145-55.

39. Villela W. Homem que é homem também pega AIDS? In: Arilha M, Ridenti GU, Medrado B, organizadores. Homens e masculinidades. Outras palavras. São Paulo: Editora 34; 1998. p. 129-42.

40. Lowndes CM. Doenças sexualmente transmissíveis na mulher. In: Giffin $\mathrm{K}$, Costa $\mathrm{H}$, organizadores. Questões da saúde reprodutiva. Rio de Janeiro: Editora Fiocruz; 1999. p. 253-80.

41. Arilha M. Homens, saúde reprodutiva e gênero: o desafio da inclusão. In: Giffin K, Costa H, organizadores. Questões da saúde reprodutiva. Rio de Janeiro: Editora Fiocruz; 1999. p. 455-67.

42. Paiva V. Sexualidades adolescentes: escolaridade, gênero e sujeito sexual. In: Parker R, Barbosa RM, organizadores. Sexualidades brasileiras. Rio de Janeiro: Relume Dumará; 1996. p. 213-36.

43. Arilha M. Homens entre a "zoeira" e a "responsabilidade”. In: Arilha M, Ridenti SGU, Medrado B, organizadores. Homens e masculinidades. Outras palavras. São Paulo: Editora 34; 1998. p. 51-78.

44. Kalckmann S. Incursões ao desconhecido: percepções de homens sobre saúde reprodutiva $\mathrm{e}$ sexual. In: Arilha M, Ridenti SGU, Medrado B, organizadores. Homens e masculinidades. Outras palavras. São Paulo: Editora 34; 1998. p. 79-100.

45. Gogna M. Factores psicosociales y culturales em la prevención y tratamiento de las enfermedades de transmisión sexual. Cad Saúde Pública 1998; 14 Supl 1:81-5.

46. Monteiro S. Gênero, sexualidade e juventude numa favela carioca. In: Heilborn ML, organizador. Sexualidade: o olhar das ciências sociais. Rio de Janeiro: Jorge Zahar; 1999. p. 117-45.

47. Paiva V, Bugamelli L, Leme B, Ventura-Filipe E, Tunala L, Santos N. A vulnerabilidade das mulheres ao HIV é maior por causa dos condicionantes de gênero? http://www.usp.br/nepaids (acessado em 24/Abr/2003).

Recebido em 30/Dez/2002

Versão final reapresentada em 20/Mai/2003

Aprovado em 28/Out/2003 